\title{
Vitamin D insufficiency and the blunted PTH response in established osteoporosis: the role of magnesium deficiency
}

\author{
O. Sahota • M. K. Mundey • P. San • I. M. Godber • \\ D. J. Hosking
}

Published online: 27 September 2006

(C) International Osteoporosis Foundation and National Osteoporosis Foundation 2006

The online version of the original article can be found at: http://dx.doi. org/10.1007/s00198-006-0084-3.

\section{O. Sahota $(\bowtie)$}

Department of Health Care of the Elderly,

Queens Medical Centre, University Hospital,

B Floor South Block,

Nottingham NG7 2UH, UK

e-mail: opinder.sahota@qmc.nhs.uk

\section{K. Mundey}

School of Biomedical Sciences,

Queens Medical Centre, University Hospital,

Nottingham, UK

P. San · D. J. Hosking

Division of Mineral Metabolism,

City Hospital,

Nottingham, UK

I. M. Godber

Clinical Laboratories,

Wishaw General Hospital,

Wishaw, Lanarkshire, UK
Due to errors on the part of the typesetter, the article was published with incorrect versions of the column heads of Table 1 and the illustration and legend of Fig. 2. The correct table and figure are given in full here. 
Table 1 Baseline demographics (mean \pm SD) $[* \mathrm{p}<0.05]$

\begin{tabular}{llll}
\hline & $\begin{array}{l}\text { Vitamin D insufficiency } \\
(25 \mathrm{OHD} \leq 30, \\
\mathrm{PTH}>57)\end{array}$ & $\begin{array}{l}\text { Functional hypoparathyroidism } \\
(25 \mathrm{OHD} \leq 30, \\
\mathrm{PTH} \leq 57)\end{array}$ & $\begin{array}{l}\text { Vitamin D replete } \\
(25 \mathrm{OHD}>30, \\
\text { PTH } 12-72)\end{array}$ \\
\hline Age $(\mathrm{yr})$ & $67.1(7.7)$ & $67.8(9.8)$ & $66.4(6.9)$ \\
BMI $\left(\mathrm{kg} / \mathrm{m}^{2}\right)$ & $27.2(3.6)$ & $26.8(3.0)$ & $26.1(4.1)$ \\
Dietary vitamin D intake $(\mathrm{mcg})$ & $2.9(4.1)$ & $2.8(3.3)$ & $3.1(2.9)$ \\
Dietary calcium intake $(\mathrm{mg})$ & $924.5(314.6)$ & $984.3(399.2)$ & $921.9(401.1)$ \\
Dietary magnesium intake $(\mathrm{mg})$ & $321.1(145.9)$ & $305.9(165.7)$ & $316.2(135.4)$ \\
Creatinine clearance $(\mathrm{lab} \mathrm{ref} 70-120 \mathrm{ml} / \mathrm{min})$ & $71.2(10.3)$ & $74.6(11.4)$ & $73.2(9.8)$ \\
Calcium $(2.2-2.6$ mmol/l) & $2.40(0.05)$ & $2.33(0.07)$ & $2.45(0.07)$ \\
Total alk. phosphatase $(40-120 \mathrm{IU} / \mathrm{L})$ & $151.9(54.5)$ & $109.5(36.2)$ & $105.2(31.7)$ \\
Magnesium $(\mathrm{lab}$ ref $0.8-1.2 \mathrm{mmol} / \mathrm{l})$ & $0.8(0.03)$ & $0.8(0.02)$ & $0.8(0.02)$ \\
Phosphate $(\mathrm{lab}$ ref $0.8-1.2 \mathrm{mmol} / \mathrm{l})$ & $1.1(0.2)$ & $1.0(0.1)$ & $1.0(0.2)$ \\
Spine BMD $\left(\mathrm{g} / \mathrm{cm}^{2}\right)$ & $0.82(0.13)$ & $0.84(0.17)$ & $0.85(0.19)$ \\
Total hip BMD $\left(\mathrm{g} / \mathrm{cm}^{2}\right)$ & $0.69(0.11)^{*}$ & $0.74(0.10)^{*}$ & $0.77(0.09)$ \\
\hline
\end{tabular}

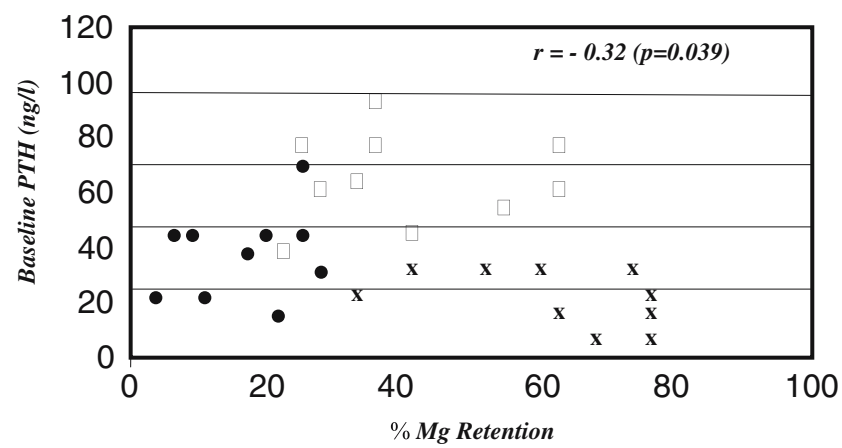

Fig. 2 Relationship between parathyroid hormone (PTH) and retention of magnesium $(\mathrm{Mg})$ following $\mathrm{Mg}$ loading in patients with low vitamin D ( $\square$ vitamin D insufficiency, $\mathbf{x}$ functional hypoparathyroidism, vitamin $\mathrm{D}$ replete) 